\title{
Obesity no barrier to knee replacement surgery
}

Published online: o3 September 2012

(C) Springer Healthcare 2012

medwireNews: Health policy should not withhold total knee arthroplasty (TKA) from patients on the basis of obesity, say UK researchers.

Data for 13,673 primary TKAs in patients with osteoarthritis performed between May 2008 and September 2010 in the UK showed that increasing body mass index (BMI) was associated with lower patient-reported measure scores both before and 6 months after surgery.

However, there was a positive trend between increasing BMI and likelihood of improvement at 6 months on patient-reported measures.

Moreover, there was no significant difference in the magnitude of improvement for patients with a morbidly obese BMI of $40-60 \mathrm{~kg} / \mathrm{m}^{2}$ and those with a healthy BMI of $15-24.9 \mathrm{~kg} / \mathrm{m}^{2}$ for the Oxford Knee Score, the EuroQoL (EQ)-5D index, and the EQ-5D visual analog scale, after adjustment for age, gender, general health and comorbidity, and American Society of Anesthesiologists grade.

“Obese patients gained as much benefit from knee replacement as patients with a 'normal' BMI, even if they did not end up at a similar postoperative level," report Paul Baker (Newcastle University) and co-workers in the Journal of Bone and Joint Surgery.

Although patients with obese $\left(\mathrm{BMI}=25-29.9 \mathrm{~kg} / \mathrm{m}^{2}\right)$ and morbidly obese BMIs had a significantly higher rate of wound complications than those with a healthy BMI (17 and 12 vs 9\%), rates of bleeding, readmission, and reoperation did not significantly differ.

While acknowledging reports indicating shorter implant survival rates in morbidly obese than healthy BMI patients, the team emphasizes that the value of TKA is linked to patient-reported gains in the quality of life

"If patients are fully informed of the elevated risks for equivalent improvements in patientreported outcome measures, then these factors should not, in themselves, be a reason to withhold surgery,” Baker et al write.

They conclude: "The decision to proceed with total knee arthroplasty should be made by the treating orthopaedic surgeon and the patient after careful consideration of all aspects of the case and the likely impact of surgery at an individual level.”

By Lynda Williams, Senior medwireNews Reporter

\section{Reference}

J Bone Joint Surg Am 2012; 94: 1501-1508 